\title{
Zwischen Notrettung und Rütlischwur: der Umbau der Wirtschafts- und Währungsunion in der Krise
}

\author{
Ulrich Hufeld*
}

Diese Abkürzungen muss man sich merken: ESM und ESS. ,ESM` steht für „European Stability Mechanism“ - Europäischer Stabilitätsmechanismus; ,ESS“ für „ESM stability support", die Stabilitätshilfe des ESM. Alles spricht dagegen, diese Begriffe als esoterische abzutun und den Spezialisten zu überlassen. Mit dem ,Rettungsschirm“ verändert sich die Wirtschafts- und Währungsunion (WWU) in den Grundfesten. Sein Inkrafttreten zum 1. Januar $2013^{1}$ vorausgesetzt, bewirkt der permanente ESM² einen Umbau der Statik: Auf dass die WWU unter allen Umständen Stabilitätsunion bleibe, verwandelt sie sich notfalls in eine Kredit- und Stabilisierungsunion. Dafür muss sich interessieren, wer mit dem Euro bezahlen, den Euro einlösen will. „Das Einlösungsvertrauen stützt sich in Zukunft nicht mehr auf die staatlich verfaßte Rechtsgemeinschaft der Bundesrepublik Deutschland, sondern wird von einer anderen Rechtsgemeinschaft und der sie stützenden Wirtschaftskraft getragen. “3 Das war die Zukunftsperspektive im März 1998, als das Bundesverfassungsgericht (BVerfG) die Verfassungsbeschwerden gegen die Teilnahme Deutschlands an der Währungsunion als ,offensichtlich unbegründet“4 verwarf. Diese Zukunft ist jetzt Gegenwart der anderen Rechtsgemeinschaft sind wir schicksalhaft verbunden.

Der ESM soll Rückhalt finden in einem neuen Art. 136 Abs. 3 des Vertrages über die Arbeitsweise der Europäischen Union (AEUV), in ganzen zwei Sätzen: „Die Mitgliedstaaten, deren Währung der Euro ist, können einen Stabilitätsmechanismus einrichten, der aktiviert wird, wenn dies unabdingbar ist, um die Stabilität des Euro-Währungsgebiets insgesamt zu wahren. Die Gewährung aller erforderlichen Finanzhilfen im Rahmen des Mechanismus wird strengen Auflagen unterliegen. " 5 Wir nähern uns der Bedeutung dieser Sätze, indem wir ihr Zustandekommen rekonstruieren als Vertrags- und Verfassungsänderung, die Risiken einträgt, wenn sie gelingt, und noch mehr Risiken, wenn sie scheitert.

- Der ESM antwortet auf die Krise, genauer: auf die Verkettung der Krisen. Die Weltfinanzkrise von 2008 hat in einzelnen Staaten der Eurozone die Verschuldungskrise verschärft und eine Staatsfinanzierungskrise heraufbeschworen mit Rückwirkung auf ,die Stabilität des Euro-Währungsgebiets“. Die Antwort, die der ESM gibt, beruht auf der Formel Soli-

* Prof. Dr. iur. Ulrich Hufeld, Inhaber des Lehrstuhls für Öffentliches Recht und Steuerrecht an der HelmutSchmidt-Universität/Universität der Bundeswehr Hamburg.

1 Auf dieses Datum zielt der Europäische Rat: Europäischer Rat 24./25. März 2011. Schlussfolgerungen, EUCO 10/11, S. 20.

2 Der permanente ESM folgt der provisorischen EFSF, der Europäischen Finanzstabilisierungsfazilität (European Financial Stability Facility). Unter dieser Firma legt eine luxemburgische Zweckgesellschaft bis 2013 Darlehensprogramme auf, verbürgt von den Mitgliedstaaten der Eurozone. Das EFSF-Volumen (effektiv 500 Milliarden Euro) übertrifft deutlich das von der Union selbst verbürgte Volumen von 60 Milliarden Euro im Europäischen Finanzstabilisierungsmechanismus (European Financial Stability Mechanism, EFSM). Näheres zum EFSM/EFSF-Provisorium siehe unten im Text.

BVerfGE 97, 350, S. 372 - Euro.

Ebenda, S. 368.

5 Europäischer Rat: 24./25. März 2011, S. 21. Vgl. Deutscher Bundestag: Drucksache 17/4880 zur Einvernehmensherstellung (Art. 23 Abs. 3 GG i.V.m. $\$ 10$ EUZBBG) von Bundestag und Bundesregierung zur Ergänzung von Art. 136 AEUV. 
darität gegen Austerität. Seine notrechtliche Rationalität ist die der temporalen Verfassungsdurchbrechung, die von der Normalordnung abweicht, um sie zu retten.

- Der ESM stellt neuerlich die Reformfrage auf der Ebene des Primärrechts, kurze Zeit schon nach dem Inkrafttreten des Vertrages von Lissabon. Jetzt muss sich erweisen, ob der neue Vertrag vorgesorgt hat oder ob sich der alte Lehrsatz bewahrheitet, dass eine allzu starre, auf Unveränderlichkeit bedachte Verfassung an ihrer anachronistischen Sturheit scheitert. Mit dem projektierten Art. 136 Abs. 3 AEUV beginnt der Post-Lissabon-Prozess. Identität und Variabilität der Verfassung: ${ }^{6}$ Dem ewigen Problem entkommt auch die Verfassung der Union nicht. Wer sie ändert, greift aber auf die europäische Gesamtverfassung zu, auf das „Gleichgewicht der politischen Kräfte Europas zwischen den Mitgliedstaaten und der Unionsebene". ${ }^{7}$ Europäische Integration und staatsverfassungsrechtliche Revision gehen Hand in Hand. ${ }^{8}$

- Die Rückkopplung ins staatliche Recht ruft in Deutschland den schillernden Begriff der Integrationsverantwortung auf. Sein harter Kern ist der absolute Parlamentsvorbehalt für jeden Vorgang der verfassungsändernden Vertragsänderung. Der Parlamentsvorbehalt blockiert von vornherein jedwede Selbstermächtigung europäischer Exekutiven. Sei es ein Brüsseler Rat oder sei es ein ESM-Gouverneursrat in Luxemburg - ohne Ermächtigung droht die Verletzung der staatlich-parlamentarischen Integrationsverantwortung aus Art. 23 Abs. 1 Grundgesetz (GG). Daraus erklärt sich die Schlagzeile der Frankfurter Allgemeinen Zeitung vom 18. Dezember 2010: „Ein Brüsseler Gipfel für die Karlsruher Richter". 9

- Der ESM wirft ein grelles Licht auf das Phänomen der Revision durch Integration. Nie war deutlicher, dass EU-Vertragsänderungen zurückwirken auf das mitgliedstaatliche Verfassungsrecht. Der ESM setzt ein neues Fundamentaldatum für die Mitglieder der Eurozone. In deren Finanzverfassungen geht ein Solidaritätsimperativ ein - mit innenpolitischen Risiken für alle Akteure: Geber müssen für Solidarität, Nehmer für Austerität werben. ${ }^{10}$ Zur Diskussion steht auch, ob der ESM den Parlamentsvorbehalt durchkreuzen und den ESS im konkreten Rettungsfall allein den Finanzministern anvertrauen darf.

- Indem aber der künftige, permanente ESM eine primärrechtliche Basis findet, drängt sich die Frage auf, welches (Notstands-)Recht die gegenwärtigen und befristeten Stabilisierungs-Mechanismen (EFSM und EFSF) trägt. Wenn Unionsorgane oder hybride Stabilisierungsstellen derzeit vorläufig-provisorische Rettungspolitik betreiben, „laufen sie Gefahr, das vorherbestimmte Integrationsprogramm zu überschreiten und außerhalb ihrer Ermächtigung zu handeln. Sie bewegen sich auf einem Pfad, an dessen Ende die Verfügungsgewalt über ihre vertraglichen Grundlagen steht"11. Haben wir mit Rettungspolitik ultra vires zu tun oder mit einem Brückenbau aus der alten in die neue WWU?

6 Statt vieler Gerd Roellecke: Identität und Variabilität der Verfassung, in: Otto Depenheuer/Michael Grabenwarter (Hrsg.): Verfassungstheorie, Tübingen 2010, § 13.

7 BVerfGE 123, 267, S. 365 - Lissabon.

8 Ausführlich zur Verkopplung von Integration durch Revision - Revision durch Integration: Ulrich Hufeld: Europäische Integration und Verfassungsänderung, in: Andreas von Arnauld/Ulrich Hufeld (Hrsg.): Systematischer Kommentar zu den Lissabon-Begleitgesetzen, Baden-Baden 2011, 1. Abschnitt, Rdz. 3-21.

9 Nikolas Busse: Ein Brüsseler Gipfel für die Karlsruher Richter, in: Frankfurter Allgemeine Zeitung, 18.12.2010.

10 Heinrich Schneider: Europas Identität (gestern) - Europas Krise (heute): ein Themenwechsel als Indiz für einen Paradigmenwechsel?, in: integration 1/2011, S. 42-62, hier S. 49-50, auch über den Zusammenhang von Solidarität und Identität.

11 BVerfGE 123, 267, S. 352 - Lissabon. 
- Am Ende steht die Prognose, dass die angekündigten Verfassungsbeschwerden jedenfalls gegen die vertrags- und verfassungsändernde Gewalt nichts ausrichten werden. Allenfalls werden sie den Bundesfinanzminister als Akteur im Gouverneursrat des ESM in parlamentarische Abhängigkeit bringen. Das wäre kein geringer Erfolg der Verfassungsbeschwerdeführer. ${ }^{12}$ Sollte aber die Ergänzung des Art. 136 AEUV scheitern, wird der provisorisch-freihändig aufgespannte Rettungsschirm nicht mehr lange zu halten sein.

\section{Der finanzpolitische Rütlischwur: Solidarität gegen Austerität}

In den Hauptstädten Europas will sich niemand auf eine weitere große Vertragsreform einlassen. Der Kraftakt von Lissabon hat den Verfassungsehrgeiz der ,Laeken-Generation “13 vollständig aufgezehrt. Die Sorge der Regierungen seit Mai 2010, dass die Notrettung qua Kredit ihrerseits in Not geraten könnte - als Instrument ohne Kompetenz, als Maßnahme ultra vires -, lenkte alle Aufmerksamkeit auf Art. 48 Abs. 6 EUV: ${ }^{14}$ das vereinfachte Änderungsverfahren. Der verschlankte Änderungsmodus soll den Befreiungsschlag ermöglichen, dem Kreditmechanismus die dringend benötigte Rechtssicherheit zuführen, zugleich aber die politischen Risiken der Vertragsänderung minimieren. Allerdings sperrt er jedwede „Ausdehnung der der Union im Rahmen der Verträge übertragenen Zuständigkeiten“. ${ }^{15}$ Unverkennbar hat sich diese Sperrklausel auf den Entwurf für einen neuen Art. 136 Abs. 3 AEUV und die Konstruktion des ESM ausgewirkt. Das Europäische Parlament sieht in der „Einrichtung des ständigen Stabilitätsmechanismus außerhalb des institutionellen Rahmens der EU eine Gefahr für die Integrität des vertragsgestützten Systems“. ${ }^{16}$

\section{Über Art. 48 Abs. 6 EUV „,zu einem neuen Modell europäischen Regierens“?}

Das Verfahren des Art. 48 Abs. 6 EUV ${ }^{17}$ kommt ohne Konvent aus, ohne Regierungskonferenz, in seiner Nomenklatur auch ohne Ratifikation. Der vereinfachte Modus setzt lediglich einen Änderungsbeschluss des Europäischen Rates voraus, die Anhörung anderer Unionsorgane $^{18}$ - dann aber die ,Zustimmung der Mitgliedstaaten im Einklang mit ihren jeweiligen verfassungsrechtlichen Vorschriften". ${ }^{19}$ So obliegt es den Staaten, die Vereinfachungsabsicht der Norm aufzugreifen und in das staatliche Europaverfassungsrecht hinein

12 Bereits anhängig sind Verfassungsbeschwerden gegen die „Griechenland-Hilfe“ und den provisorischen „Rettungsschirm“; vgl. die Eilentscheidung BVerfGE 125, 385.

13 Zur Einberufung des Europäischen Verfassungskonvents in Laeken 2001 statt vieler Thomas Oppermann: Konventsmethode und ,gemischte“ Entstehung der Unionsverfassung, in: Klaus Beckmann/Jürgen Dieringer/Ulrich Hufeld (Hrsg.): Eine Verfassung für Europa, 2. Aufl., Tübingen 2005, S. 59-82, hier S. 63; Hartmut Marhold: Von der Währungsunion zur (gescheiterten) Konstitutionalisierung der Europäischen Union - zehn Jahre Systemreform, ein Kapitel Integrationsgeschichte (1999-2008), in: integration 1/2011, S. 3-23, hier S. 8-15.

14 Vertrag über die Europäische Union.

15 Art. 48 Abs. 6 UAbs. 3 EUV.

16 Europäisches Parlament: Legislative Entschließung des Europäischen Parlaments vom 23. März $2011 \mathrm{zu}$ dem Entwurf eines Beschlusses des Europäischen Rates zur Änderung des Artikels 136 des Vertrags über die Arbeitsweise der Europäischen Union hinsichtlich eines Stabilitätsmechanismus für die Mitgliedstaaten, deren Währung der Euro ist, P7_TA(2011)0103, S. 5.

17 Sein Anwendungsbereich beschränkt sich - erstreckt sich - auf den umfänglichen dritten Teil des AEUV: „Die internen Politiken und Maßnahmen der Union“, Art. 26-197, darunter die Wirtschafts- und Währungspolitik, Art. 119-144. Ausführlich zu Art. 48 Abs. 6 EUV Hannes Rathke: Materielle Vertragsänderungen, in: Andreas von Arnauld/Ulrich Hufeld (Hrsg.): Systematischer Kommentar zu den Lissabon-Begleitgesetzen, Baden-Baden 2011, 6. Abschnitt, Rdz. 28-35.

18 Art. 48 Abs. 6 UAbs. 2 Satz 2 EUV: Anhörung des Europäischen Parlaments und der Kommission, ,bei institutionellen Änderungen im Währungsbereich“" auch der Europäischen Zentralbank (EZB).

19 Art. 48 Abs. 6 UAbs. 2 Satz 3 EUV. Vgl. demgegenüber den mitgliedstaatlichen Ratifikationsvorbehalt des Art. 48 Abs. 4 UAbs. 2 EUV (Ordentliches Änderungsverfahren). 
zu verlängern. Für Deutschland steht die Verknüpfung des Art. 48 Abs. 6 EUV mit der Integrationsgesetzgebung der einfachen Zustimmungsmehrheiten in Bundestag und Bundesrat zur Diskussion. ${ }^{20}$ Und Irland mag Gründe finden, die obligatorische Volksabstimmung als Ratifikationsvoraussetzung für, große " Vertragsreformen im ordentlichen Änderungsverfahren $^{21} \mathrm{zu}$ reservieren. Dann hätte Art. 48 Abs. 6 EUV eine Bresche geschlagen für ,kleine“ Reformen, die ungestört parlamentarisch, unionsweit ohne Referendum gebilligt werden können; wohlweislich hat Bundeskanzlerin Angela Merkel stets verlauten lassen, für die Befestigung des Rettungsschirms genüge eine ,eng begrenzte Vertragsänderung“. ${ }^{22}$

Art. 48 Abs. 6 EUV eröffnet einen unauffälligeren Zugriff auf das primäre Unionsrecht. Seine Sperrklausel ${ }^{23}$ blockiert aber hartnäckig Kompetenzzuwächse auf seiten der Union. Die von der , ordentlichen “ abgestufte, also relativ , vereinfachte“ Vertragsänderung sperrt sich gegen die „Übertragung von Hoheitsrechten“ - gegen jenen Grundtatbestand der Integration, der Anlass gegeben hat, Integrationsgesetzgebung als Verfassungsänderung zu begreifen, als Begründung von Hoheitsgewalt außerhalb der Staatsorganisation. Allein diese Ausgründung verändert die Verfasstheit der integrierten Staaten, die Kompetenzen übertragen. Eben diese Öffnung verlangt die Verfasstheit der ausgegründeten Hoheitsgewalt. So bewirkt der Kompetenztransfer durch Vertrag regelmäßig eine doppelte Verfassungsänderung, Zuständigkeitsverlust auf seiten der Staaten und Kompetenzzuwachs auf seiten der Union. Wie aber deuten wir eine Vertragsänderung, die im Modus des Art. 48 Abs. 6 EUV den Kompetenztransfer vermeiden muss? Geht es hier um Vorstufen der Integration, um Elemente zwischenstaatlicher Zusammenarbeit, die - wie vormals die Gemeinsame Außenund Sicherheitspolitik (GASP) und die Polizeiliche und Justizielle Zusammenarbeit in Strafsachen (PJZS) - noch keinen Platz finden im Zentrum der Supranationalität?

$\mathrm{Zu}$ diesen Fragen bietet der ESM ein historisches Lehrstück. Ersichtlich fehlt ihm die Eigenständigkeit, wie sie kennzeichnend war für die ,Säulen“ in der Tempelstruktur vor ,Lissabon'. Konstruiert als zwischenstaatliche Einrichtung, schiebt er sich als Zweckbau in die asymmetrische Statik der WWU mit staatlicher Fiskal- und supranationaler Währungspolitik. Der ESM bleibt der Supranationalisierungssperre des Art. 48 Abs. 6 EUV verpflichtet, bewirkt aber einen Umbau der europäischen Wirtschafts- und Währungsverfassung. Mit ihm wird ,der Charakter der bestehenden Währungsunion grundsätzlich geändert“ ${ }^{24}$ Der Europäische Rat kann nicht verschleiern, dass er die WWU-Statik verschieben will. Aus Herkommen ${ }^{25}$ und Stoßrichtung - Abkehr von der WWU in der unvollkommenen Gestalt von ,Maastricht ‘ - erklärt sich der eigentümliche Bauplan des ESM. Der neue WWU-Imperativ will den ESM aktiviert sehen, ,wenn dies unabdingbar ist, um die Stabilität des Euro-Währungsgebiets insgesamt zu wahren“, verpflichtet damit auf ein supranationales Unionsziel und ermächtigt zum intergouvernementalen Handeln.

$20 \S 2$ des Integrationsverantwortungsgesetzes (IntVG) hilft nicht weiter, verlangt deklaratorisch und unspezifisch ,ein Gesetz gemäß Artikel 23 Absatz 1 des Grundgesetzes“. Näher zum Problem - einfache Mehrheiten (Art. 23 Abs. 1 Satz 2 GG) oder Zweidrittelmehrheiten (Art. 23 Abs. 1 Satz 3 GG) - unten im Text.

21 Art. 48 Abs. 2-4 EUV.

22 Deutscher Bundestag, 17. Wahlperiode, Stenografischer Bericht der 67. Sitzung, 27. Oktober 2010, Plenarprotokoll 17/67, S. 7084 (A).

23 Art. 48 Abs. 6 UAbs. 3 EUV.

24 Otmar Issing: Gefahr für die Stabilität, in: Frankfurter Allgemeine Zeitung, 11.11.2010, S. 14, freilich noch nicht im konkreten Bezug zum ESM, sondern allgemein für ,die Aufgabe beziehungsweise Einschränkung des No-Bail-Out-Prinzips“. Jürgen Habermas: Ein Pakt für oder gegen Europa?, in: Süddeutsche Zeitung, 07.04.2011, sieht in den Gipfelbeschlüssen vom 24./25. März 2011 einen ,expertokratisch ausgehandelten, in den Wirtschaftsteilen der Presse versickerten, fast tonlos vollzogenen Politikwechsel zu einer intensiveren Abstimmung von Politiken, die gemäß dem Europavertrag in nationale Zuständigkeit fallen“.

25 Art. 48 Abs. 6 EUV - gleichsam im Schatten der großen Vertragsreformpolitik. 
Das Europäische Parlament reagiert besorgt, will die Unionsorgane in den ESM eng eingebunden sehen und ,,unterstreicht, dass der Mechanismus nicht zu einem neuen Modell europäischen Regierens führen sollte“: Art. 48 Abs. 6 EUV müsse „Ausnahmeverfahren“ bleiben, ,zu einem geeigneten Zeitpunkt“ müsse die „Methode der Union“" greifen und der ESM ein ,Mechanismus der Union werden“. ${ }^{26}$ Hier vernachlässigt das Europäische Parlament allerdings, dass der ESM von staatlicher Finanzkraft lebt und auf die Bereitschaft baut, in der Stunde der Not das staatliche Finanzverfassungsrecht zu durchbrechen.

\section{Dreifache Verfassungsdurchbrechung}

Jede bündische Finanzverfassung oszilliert zwischen Autonomie und Solidarität. Die WWU in der Fasson von ,Maastricht‘ hält das Autonomieprinzip hoch. Dafür steht vor allem das ,bail out'-Verbot. ${ }^{27}$ Die Gemeinschaft der Zwölf wollte 1992 eine asymmetrische Wirtschafts- und Währungsunion mit staatlich kontrollierter Haushalts- und Wirtschaftspolitik im Rahmen einer Koordinierung ${ }^{28}$ und vergemeinschafteter Geldpolitik. Mit der Souveränität in der Fiskalpolitik ${ }^{29}$ korrespondierte die mit ,no bail out ' festgeschriebene Vereinzelung: Aus der Alleinverantwortlichkeit für den Staatshaushalt ergab sich die Alleinverantwortlichkeit für den Umfang der Kredite auf der Einnahmenseite und die Kreditfinanzierungskosten. Die ,no bail out'-Regel wollte nicht nur ,moral hazard' in der Haushaltswirtschaft ausschließen, sondern mit der Alleinstellung auf dem Kapitalmarkt ein staatenindividuelles Alarmsystem gewährleisten. ${ }^{30}$ Einzelstaatenbezogene Risikoaufschläge hätten im Verein mit einem kräftigen Stabilitäts- und Wachstumspakt alle Staaten davon abhalten müssen, ihre Zahlungs- und Kreditfähigkeit zu gefährden. Das Konzept vertraute auf präventive Komponenten ${ }^{31}$ - und traf keine Vorsorge für den Fall, dass die Koordinierung der Wirtschaftspolitik und das Recht der Defizitbegrenzung scheitern und die Refinanzierungsnot eines Mitgliedstaats der Eurozone nicht verhindern können. Die besondere Notstandsklausel des Art. 122 Abs. 2 AEUV belegt in ihrer Beschränkung auf katastrophale und unkontrollierbare Schwierigkeiten, dass das Präventivkonzept ein allgemeines Hilferecht gerade erübrigen wollte.

Eben dafür, für ein allgemeines (Not-)Hilferecht will der geplante Art. 136 Abs. 3 AEUV eine Ermächtigungsgrundlage anbieten. Das ist eine konstitutive Änderung der Unionsverfassung. Das bisherige (noch gültige) Vertragsrecht hat die subsidiäre und konditionierte Staatenhilfe qua ESS weder erlaubt noch verboten; es wollte ihr zuvorkommen und ist damit gescheitert. Jetzt verschiebt sich die Achse in der europäischen Finanzverfassung in Richtung ,Solidarität zwischen den Mitgliedstaaten“. ${ }^{32}$ Im Krisenfall gilt nicht mehr das Autonomieprinzip. An seine Stelle tritt Solidarität gegen Austerität, finanzieller Beistand gegen budgetäre Enthaltsamkeit, Kredit gegen Wohlverhalten. Die Eurozone übernimmt die im In-

26 Europäisches Parlament: Legislative Entschließung des Europäischen Parlaments vom 23. März 2011, S. 5, 7, 9.

27 Art. 125 AEUV.

28 Vgl. Art. 5 Abs. 1 und Art. 2 Abs. 5 AEUV zur Kompetenz-Kategorie „Koordinierung“.

29 Hans Tietmeyer: Währungsstabilität - auch in Europa gefordert, in: Paul Kirchhof/Klaus Offerhaus/Horst Schöberle (Hrsg.): Steuerrecht, Verfassungsrecht, Finanzpolitik. Festschrift für Franz Klein, Köln 1994, S. 3342, hier S. 39.

30 Stefan Pilz/Heidi Dittmann: Die Europäische Wirtschafts- und Währungsunion am Scheideweg, in: Die Öffentliche Verwaltung 11/2011, S. 438-447, hier S. 447, verweisen auf moral hazard-Verhalten seitens privater Gläubiger, dem mit einem Insolvenzrecht für Staaten begegnet werden könne; zum Spekulationsargument Dirk Meyer: Destabilisierende Spekulation als Rechtfertigung eines Europäischen Stabilitätsmechanismus?, in: Wirtschaftsdienst 6/2011, S. 391-397.

31 Kai von Lewinski: Öffentlichrechtliche Insolvenz und Staatsbankrott, Tübingen 2011, S. 439-443.

32 Art. 3 Abs. 3 UAbs. 3 EUV. 
ternationalen Währungsfonds (IWF) erprobte Mechanik einer Hilfe im Zeichen der Konditionalität - ,Washington Consensus conditionalities ${ }^{633}$-, stellt sie aber in einen einheitlichen Währungsraum und verändert den bündischen Verfassungskontext. Diesen Kontext verkennt, wer den ESM als bloße Fortsetzung eines außervertraglichen Krisenmanagements ${ }^{34}$ begreift.

Die Geberstaaten übernehmen im ESM eine Wiederherstellungsverantwortung. Eine markante Ausprägung dieser Zielverpflichtung findet sich in der EFSM-Verordnung vom 11. Mai 2010. ${ }^{35}$ Deren synallagmatische Logik macht den Zugriff der Sicherungsgeber auf die fiskalpolitische Souveränität des Kreditnehmers begreiflich. Das Darlehen wird verkoppelt mit ,Bedingungen“, um ,eine solide wirtschaftliche oder finanzielle Situation in dem begünstigten Mitgliedstaat und dessen eigene Finanzierungsfähigkeit auf den Finanzmärkten wiederherzustellen“. ${ }^{36}$ In dieser Lage der Selbstentmachtung auf Antrag verständigen sich die Beteiligten (EFSM/EFSF, künftig ESM, und der , begünstigte` Mitgliedstaat) auf eine Flucht aus der Koordinierung: Die souveränitätsschonende Kompetenz-Kategorie ${ }^{37}$ wird für die Laufzeit des Darlehens überschrieben - Musterfall einer temporalen Verfassungsdurchbrechung, die eine reguläre Ordnung notrechtlich und befristet außer Geltung setzt, gerichtet auf Wiederherstellung. „Wenn dies unabdingbar ist“, so der neue Art. 136 Abs. 3 AEUV, wird das ESM-Recht ,aktiviert“" als außerordentliches WWU-Recht, um Rettung und Rückkehr zum ordentlichen WWU-Recht in der Fasson von ,Maastricht' bemüht. Außerordentlich übernimmt der ESM Regierungsfunktionen und der , begünstigte` Mitgliedstaat verliert als Bittsteller die Hoheit über seine Steuer- und Budgetpolitik.

Der ESS hat das Format einer verfassungsdurchbrechenden Maßnahme im Sinne der Kontrastbegrifflichkeit Carl Schmitts. ,Solche Durchbrechungen sind ihrer Natur nach Maßnahmen, keine Normen [...]. Ihre Notwendigkeit ergibt sich aus der besonderen Lage eines Einzelfalles, aus einer unvorhergesehenen abnormen Situation. Wenn im Interesse des Ganzen solche Durchbrechungen und Maßnahmen vorgenommen werden, so zeigt sich darin die Überlegenheit des Existenziellen über die bloße Normativität. Wer zu solchen Handlungen befugt und imstande ist, handelt souverän. " ${ }^{38}$ Von dieser Souveränität profitieren gegenwärtig Griechenland, ${ }^{39}$ Irland und Portugal. Und der projektierte Art. 136 Abs. 3 AEUV steht für den Versuch, aus der Unterlegenheit der Normativität herauszufinden, der Maßnahme eine normative Grundlage zu geben - und aus allzu souveräner Solidarität einen unionsrechtlichen Solidaritätsimperativ zu formen. Die geschriebene und begründete, definierte, damit begrenzte und kontrollierbare Verfassungsdurchbrechung ist die verfassungsnormativ

33 Sabine Schlemmer-Schulte: Internationales Währungs- und Finanzrecht, in: Christian Tietje (Hrsg.): Internationales Wirtschaftsrecht, Berlin 2009, §9 Rdz. 52-56; Markus Krajewski: Wirtschaftsvölkerrecht, 2. Aufl., Heidelberg 2009, Rdz. 780.

34 Daniel Thym: Euro-Rettungsschirm: zwischenstaatliche Rechtskonstruktion und verfassungsgerichtliche Kontrolle, in: Europäische Zeitschrift für Wirtschaftsrecht 5/2011, S. 167-171, hier S. 167, bezogen auf den heutigen Rettungsschirm EFSM/EFSF.

35 Verordnung (EU) Nr. 407/2010 des Rates vom 11. Mai 2010 zur Einführung eines europäischen Finanzstabilisierungsmechanismus, in: Amtsblatt der EU, Nr. L 118 vom 12. Mai 2010, S. 1-4 (EFSM-VO).

36 Art. 3 Abs. 3 lit. b EFSM-VO. Vgl. Europäischer Rat: 24./25. März 2011, Anlage II, S. 21-34, hier S. 25 : „Vereinbarung über die Merkmale des ESM“, „Wiedererlangung des Zugangs zu den Finanzmärkten“.

37 Art. 2 Abs. 5 AEUV: „Unterstützung, Koordinierung oder Ergänzung der Maßnahmen der Mitgliedstaaten“.

38 Carl Schmitt: Verfassungslehre, Berlin 1928, S. 107.

39 Die Griechenland-Hilfe war zunächst ad hoc-Hilfe vor Einrichtung des EFSM/EFSF-Provisoriums. Dazu Christoph Herrmann: Griechische Tragödie - der währungsverfassungsrechtliche Rahmen für die Rettung, den Austritt oder den Ausschluss von überschuldeten Staaten aus der Eurozone, in: Europäische Zeitschrift für Wirtschaftsrecht 11/2010, S. 413-418. Zur weitreichenden Fortschreibung der Griechenland-Hilfe vgl. die Erklärung der Staats- und Regierungschefs des Euro-Währungsgebiets und der EU-Organe vom 21. Juli 2011. 
gebändigte Maßnahme. ${ }^{40}$ Diesen Rückhalt hat der ESM bitter nötig, als Krisenmechanismus im Ausnahmezustand und dreifache Verfassungsdurchbrechung:

- Der Rütlischwur dementiert das Autonomiekonzept von ,Maastricht", die Eigen- und Alleinverantwortung für das Staatsbudget - nicht in der Normallage, wohl aber in finanzpolitischer Not und Gefahr (unionsrechtliche Verfassungsdurchbrechung): Der neue Art. 136 Abs. 3 AEUV verpflichtet im Krisenfall auf das Interesse des Ganzen, auf ,die Stabilität des Euro-Währungsgebiets insgesamt“.

- Der Solidaritätsimperativ nimmt die nicht-notleidenden Staaten als Geber in die Pflicht, bürdet ihnen im Krisenfall die final supranationale, instrumentell intergouvernementale Wiederherstellungsverantwortung auf, die budgetär abgesichert werden muss. Die Finanzkraft der Geberstaaten wird anomal herausgefordert und deren Finanzverfassungen wird temporär ein supranationales Anliegen eingeschrieben (staatsrechtliche Verfassungsdurchbrechung auf seiten der Geberstaaten).

- Das Austeritätsprogramm bindet den notleidenden Staat. Er unterwirft sich - notgedrungen freiwillig - und regiert sich haushaltsverfassungsrechtlich im Ausnahmezustand (staatsrechtliche Verfassungsdurchbrechung auf seiten der Nehmerstaaten). Bis zur Wiederherstellung eigener Finanzierungsfähigkeit verschieben sich auch politisch die Koordinaten des Regierungshandelns. Die budgetär abhängige Regierung muss um das Vertrauen der Bürgerschaft in den Austeritätskurs werben; womöglich sieht sie sich bedrängt, die ,strengen Auflagen“41 nachzuverhandeln. ${ }^{42}$

Der „Durchführungsbeschluss des Rates vom 7. Dezember 2010 über einen finanziellen Beistand der Union für Irland“"43 führt exemplarisch und drastisch vor Augen, wie der verfassungsrechtliche Anspruch auf Selbstregierung zur Verhandlungsmasse im Ringen um Finanzhilfe wird. Der Beschluss gewährt ein Darlehen in Höhe von maximal 22,5 Milliarden Euro und erwartet im Gegenzug, dass Irland Jahr für Jahr einen ,spezifizierten“ Maßnahmenkatalog abarbeitet und einem bezifferten „Defizitpfad“ folgt. In den Jahren 2011 und 2012 unter anderem Erhöhung der Einnahmen durch Senkung der Einkommensteuerstufen und -vergünstigungen; Reduzierung der Steuererleichterungen für die private Altersvorsorge; Erhöhung der Kohlenstoffsteuer; Gehaltskürzung für neue Beschäftigte im öffentlichen Dienst um 10 Prozent; Erhöhung des Rentenalters; „Berechnungsgrundlage für die Renten muss das in der Dienstzeit bezogene Durchschnittsgehalt" werden; Reform des Arbeitslosenunterstützungssystems; Einsetzung eines Haushaltsbeirats; hinzu kommen konkrete Gesetzgebungsaufträge etwa zur Restrukturierung der Kreditinstitute und zur Reform des Privatschuldensystems. „Die Auszahlung jeder weiteren Rate erfolgt auf der Grundlage einer zufriedenstellenden Umsetzung des Programms“. ${ }^{4}$

40 Zum Begriff der (materiellen) Verfassungsdurchbrechung und zum Konzept der kontrollierten Ausnahme eingehend Ulrich Hufeld: Die Verfassungsdurchbrechung, Berlin 1997, S. 15-25, 208-230.

41 So im Wortlaut der geplante Art. 136 Abs. 3 Satz 2 AEUV.

42 In Gödöllő ließen die EU-Finanzminister im April 2011 verlauten, dass ,,alle portugiesischen Parteien den Auflagen zustimmen sollen“. So solle verhindert werden, dass sich die neugewählte Regierung nicht an das zuvor ausgehandelte Programm hält. Vgl. Werner Mussler/Michaela Seiser: EU schließt Umschuldung Griechenlands aus, in: Frankfurter Allgemeine Sonntagszeitung, 10.04.2011.

43 Amtsblatt der EU, Nr. L 30 vom 4. April 2011, S. 34-39. Vgl. auch Durchführungsbeschluss des Rates vom 30. Mai 2011 über einen finanziellen Beistand der Union für Portugal, in: Amtsblatt der EU, Nr. L 159 vom 17. Juni 2011, S. 88-92.

44 Art. 3 Abs. 2, Abs. 3, Abs. 7-9 Durchführungsbeschluss über einen finanziellen Beistand der Union für Irland. 


\section{Der Hybridcharakter des ESM}

Der geplante Art. 136 Abs. 3 Satz 2 AEUV erhebt das Notfallkonzept Solidarität gegen Austerität zur primärrechtlichen Vorgabe: „Die Gewährung aller erforderlichen Finanzhilfen im Rahmen des Mechanismus wird strengen Auflagen unterliegen." Die vorgeschlagene Neuregelung gibt indessen keine Auskunft über Organzuständigkeiten. Wer gewährt die Hilfen, wer überwacht die Auflagen? Satz 1 spricht die Mitgliedstaaten der Eurozone an als diejenigen Akteure, die „einen Stabilitätsmechanismus einrichten“. Der Wortlaut schließt nicht aus, dass sie sich im Mechanismus supranational formieren, zumal sie mit der Stabilität der Eurozone ein supranationales Ziel verfolgen. Jedoch steht Art. 48 Abs. 6 UAbs. 3 EUV einer Auslegung entgegen, die den ESM zum Hoheitsträger mit Durchgriffsbefugnissen macht. Die Supranationalisierungssperre verhindert auch eine Vergemeinschaftung in zweiter Linie, eine mittelbare Entstaatlichung in der operativen Durchführung des Solidaritätsimperativs.

Die Mitglieder der Union sind als Herren der Verträge die Wegbereiter, die Mitgliedstaaten der Eurozone Gründer und Träger des ESM. Er ist konzipiert als ,zwischenstaatliche Organisation nach dem Völkerrecht"“. ${ }^{45}$ Der Europäische Rat ordnet ihr in seiner Vereinbarung über die Merkmale des ESM vom 24./25. März 2011 die Aufgabe zu, „Finanzmittel zu mobilisieren [...], um die Finanzstabilität des Euro-Währungsgebiets insgesamt zu wahren“ ${ }^{46}$ Der Rückhalt der staatlichen Bürgen und ,die höchste Bonitätseinstufung der wichtigsten Kreditratingagenturen" 47 gewährleisten günstig finanzierbare ESM-Anleiheemissionen. Vorbehaltlich einer späteren „Anpassung des gezeichneten Kapitals“ verfügt er über insgesamt 700 Milliarden Euro, zusammengesetzt aus abrufbarem Kapital (80 Milliarden Euro) und Bürgschaften (620 Milliarden Euro), für eine effektive Darlehenskapazität von 500 Milliarden Euro; das zusätzliche Kapital (200 Milliarden Euro) sichert das Top-Rating. „Die Darlehenskapazität des ESM soll nach Möglichkeit durch die Beteiligung des IWF an Finanzhilfemaßnahmen ergänzt werden; daneben können sich auch Mitgliedstaaten, die dem Euro-Währungsgebiet nicht angehören, auf Ad-hoc-Basis beteiligen.“"48 Der ESM handelt nicht hoheitlich, sondern vertraglich. Das gilt für seine Anleihen, vor allem aber für den ESS. Der ESS wird ,in Form von Darlehen“ gewährt „unter strikten Auflagen gemäß einem makroökonomischen Anpassungsprogramm". ${ }^{49}$ Im ESM regiert ein Gouverneursrat (Board of Governors), dem die Finanzminister der Mitgliedstaaten der Eurozone angehören. Er ist „das höchste Beschlussfassungsorgan des ESM“.50 Die „,wichtigen Beschlüsse“ - über die Gewährung von und Bedingungen der Finanzhilfe; die Darlehenskapazität des ESM; die Änderungen des Instrumentariums - fasst er ,im gegenseitigen Einvernehmen“ “51

45 Europäischer Rat: 24./25. März 2011, Anlage II, S. 22.

46 Ebenda.

47 Ebenda, S. 23.

48 Ebenda, S. 21, 23-24 zur Kapitalstruktur.

49 Ebenda, S. 25.

50 Ebenda, S. 23.

51 Ebenda, S. 22-23, dazu S. 21, Fn. 1: „Ein im gegenseitigen Einvernehmen gefasster Beschluss ist ein Beschluss, der mit Einstimmigkeit der an der Abstimmung teilnehmenden Mitgliedstaaten gefasst wird, d.h. Stimmenthaltungen verhindern nicht die Annahme des Beschlusses." Das bedeutet für Deutschland: Ein Dissens in der Bundesregierung führt im Gouverneursrat zur Enthaltung des Bundesministers der Finanzen, hindert aber nicht die Inpflichtnahme der Bundesrepublik mit ihrem Finanzierungsanteil. Wenn und weil der haushaltsrechtliche Parlamentsvorbehalt greift, mag allenfalls im Bundestag eine negative Mehrheit zustande kommen, die den Finanzminister auf Ablehnung verpflichtet. Vgl. Koalitionsvertrag zwischen CDU, CSU und FDP, 17. Legislaturperiode, sub VI. 3.1: „Im Kabinett wird in Fragen, die für einen Koalitionspartner von grundsätzlicher Bedeutung sind, keine Seite überstimmt.“ 
Mit diesen Merkmalen und Handlungsformen präsentiert sich der ESM als zwischenstaatliche Organisation ohne eigene Hoheitsgewalt. Dennoch, die hybride Kombination aus intergouvernementalen und supranationalen Elementen ist nicht zu übersehen. Auf der operativen Ebene, bei „Aktivierung der Finanzhilfe“, wird der Kommission eine wichtige Rolle zugedacht. Sie soll mit dem IWF und in Absprache mit der EZB ,den tatsächlichen Finanzierungsbedarf des begünstigten Mitgliedstaats“ ermitteln; ermächtigt vom Gouverneursrat, verhandelt sie das Austeritätsprogramm und überwacht die „Einhaltung der politischen Auflagen", wiederum in der Troika mit IWF und EZB. ${ }^{52}$ Der Gouverneursrat wird angehalten, die ESM-Entscheidungen im ,zwischenstaatlichen Rahmen“ mit den Verfahren im „Überwachungsrahmen der EU ${ }^{“ 53}$ zu verzahnen. ${ }^{54}$ Der Hybridcharakter des ESM spiegelt freilich nur die Grundentscheidung der projektierten WWU-Ergänzung, mit einem zwischenstaatlichen Mechanismus die Stabilität in der Eurozone abzusichern. In ihrer Zielgebundenheit führt die Vertragsänderung zurück in die Herzkammer der supranationalen Union. So sehr die Sperrklausel des Art. 48 Abs. 6 UAbs. 3 EUV die intergouvernementale Konstruktion bestimmt, so offensichtlich ist der ESM teleologisch die Antwort auf eine Schicksalsfrage der supranational verfassten Schicksalsgemeinschaft.

\section{Ein Brüsseler Gipfel für die Karlsruher Richter?}

In ihrer Berichterstattung über das Treffen des Europäischen Rates im Dezember 2010 befand die Frankfurter Allgemeine Zeitung unter der Überschrift „Ein Brüsseler Gipfel für die Karlsruher Richter“: „Vielen EU-Regierungen gilt die Vertragsänderung als ein Gefallen, den sie Deutschland tun, damit Frau Merkel das Bundesverfassungsgericht nicht fürchten muss. " ${ }^{\text {55 }}$ War die Furcht begründet? Hatte nicht das BVerfG fünf Monate zuvor - mit dem Mangold/Honeywell-Beschluss ${ }^{56}$ vom Juli 2010 - das furchterregende Lissabon-Urteil $^{57}$ vom Juni 2009 ,kassiert“58 und seinen Kontrollanspruch gerade in der Grenzbelastungsprobe zurückgenommen? Warum geht die Bundesregierung das Risiko ein, mit einer als unabdingbar ausgegebenen Vertragsänderung zu scheitern, in Helsinki oder andernorts? Droht ihr Ungemach in der eigenen Hauptstadt, weil sie sich dort womöglich um Zweidrittelmehrheiten bemühen muss, im Bundestag und im Bundesrat, mithin um Zustimmung der Opposition? Und selbst wenn die qualifizierten Mehrheiten für den neuen Art. 136 Abs. 3 AEUV und den ESM zustandekommen: Hat der verfassungsändernde Integrationsgesetzge-

52 Europäischer Rat: 24./25. März 2011, S. 27. Vgl. Beschluss der Vertreter der Regierungen der Mitgliedstaaten der Europäischen Union vom 24. Juni 2011 (ECOFIN 462): „Die Vertreter der Regierungen der Mitgliedstaaten der Europäischen Union kommen überein, dass der ESM-Vertrag Bestimmungen enthalten sollte, nach denen die Europäische Kommission und die Europäische Zentralbank die in dem Vertrag aufgeführten Aufgaben ausführen."

53 Art. 121, 126, 136 Abs. 1 AEUV.

54 Europäischer Rat: 24./25. März 2011, S. 28 unter der Überschrift „Vereinbarkeit mit dem Rahmen der multilateralen Überwachung der EU“"

55 Busse: Ein Brüsseler Gipfel für die Karlsruher Richter, 2010.

56 BVerfGE 126, 286.

57 BVerfGE 123, 267. Dazu Peter-Christian Müller-Graff: Das Karlsruher Lissabon-Urteil: Bedingungen, Grenzen, Orakel und integrative Optionen, in: integration 4/2009, S. 331-360; Ulrich Hufeld: Vom Wesen der Verfassung Europas, in: Peter Häberle (Hrsg.): Jahrbuch des öffentlichen Rechts, Band 59, Tübingen 2011, S. 457-475.

58 So Joachim Wuermeling: Was bleibt noch vom Lissabon-Urteil? Kurzkommentar zur Mangold-Entscheidung des Bundesverfassungsgerichts vom 26.8.2010, abrufbar unter: www.europa-union.de/fileadmin/files_eud/ PDF-Dateien_EUD/AG_Europa-Professionell (letzter Zugriff: 30.06.2011). Dort weiter: „Denn an die Ausübung der Ultra-vires-Kontrolle, die das Lissabon-Urteil etabliert hat, und die ein zentraler Stein des Anstoßes war, werden kaum zu erreichende Anforderungen gestellt.“ 
ber freie Hand, Deutschland in eine solidarische Stabilisierungsunion zu führen? Oder hat er auf diesem Wege die Karlsruher „Identitätskontrolle“ zu fürchten?

\section{Der ESM und die „Selbstgestaltungsfähigkeit eines Verfassungsstaates“}

Das ESM-Notfall-Regime fordert die defensive Staatsaufgabenlehre des Lissabon-Urteils heraus. Mit seinem berühmten Fünf-Punkte-Katalog von Karlsruhe hat das BVerfG Schwergewichte des Politischen aufgebaut, Bollwerke gegen weitere Integration, ausgewählte Reservate staatlicher Hoheit: das Strafrecht, das staatliche Gewaltmonopol, der Sozialstaat, die Kulturhoheit - und die Finanzhoheit werden den ,wesentlichen Bereichen demokratischer Gestaltung“"59 zugeschlagen, seien ,besonders sensibel für die demokratische Selbstgestaltungsfähigkeit eines Verfassungsstaates““ ${ }^{60}$ Hier gehe es um politische Entscheidungen, „die sich im parteipolitisch und parlamentarisch organisierten Raum diskursiv entfalten". ${ }^{61}$ Für die Budgetpolitik wird das näher präzisiert: „Die Hoheit über den Haushalt ist der Ort konzeptioneller politischer Entscheidungen über den Zusammenhang von wirtschaftlichen Belastungen und staatlich gewährten Vergünstigungen. Deshalb wird die parlamentarische Aussprache über den Haushalt - einschließlich des Maßes der Verschuldung - als politische Generaldebatte verstanden. Nicht jede haushaltswirksame europäische oder internationale Verpflichtung gefährdet die Gestaltungsfähigkeit des Bundestages als Haushaltsgesetzgeber. [...] Entscheidend ist aber, dass die Gesamtverantwortung mit ausreichenden politischen Freiräumen für Einnahmen und Ausgaben noch im Deutschen Bundestag getroffen werden kann.“62

Der Katalog von Karlsruhe erfüllt zwei Funktionen: Zum einen konkretisiert er den Maßstab des Art. 79 Abs. 3 GG. Darauf kommt es europapolitisch an, weil sich die verfassungsrechtliche Gebundenheit der Integrationsgesetzgebung regelmäßig auf diese eine, so anspruchsvolle wie unbestimmte Maßstabsnorm reduziert. Der Maßstab und die deutsche Europapolitik haben sich seit den 1990er Jahren konfliktträchtig angenähert. In kontinuierlicher Integrationsabsicht findet sich der Gesetzgeber bereit, auf die Ermächtigung des Art. 23 Abs. 1 GG zurückzugreifen, Hoheitsrechte zu übertragen und an die Grenznorm heranzurücken. Indessen hat das BVerfG nicht mit einer unionsrechtskonformen Auslegung des Art. 79 Abs. 3 GG geantwortet, sondern mit etatistischer Profilierung und Kontrollverschärfung. Der Katalog von Karlsruhe erschließt mit jedem prominent herausgestellten Politikfeld bereichsspezifisch neues Potenzial für intensivierte Kontrolle. Darüber hinaus ist dem Katalog mit dem Mangold/Honeywell-Beschluss eine zweite Funktion zugewachsen: In den Bereichen der „,besonders sensiblen“ Staatsreservate gilt das mit ,Mangold/Honeywell“ begründete Prinzip der Zurückhaltung und Fehlertoleranz im Ultra-vires-Streit nicht. Im Gegenteil, der verfassungsgerichtliche Widerstand gegen ,eigenmächtige Kompetenzerweiterungen“ wird verschärft, wenn sie sich ,,auf Sachbereiche erstrecken, die zur verfassungsrechtlichen Identität der Mitgliedstaaten rechnen oder besonders vom demokratischen Prozess in den Mitgliedstaaten abhängen“.63

Dass , ,die fiskalischen Grundentscheidungen über Einnahmen und - gerade auch sozialpolitisch motivierte - Ausgaben der öffentlichen Hand" ${ }^{\text {"64 }}$ teilhaben am herausgehobenen Schutz der demokratischen Selbstgestaltungsfähigkeit im Diskursraum Staat, hatte freilich bereits das

59 BVerfGE 123, 267, S. 358 - Lissabon.

60 Ebenda, S. 359.

61 Ebenda, S. 358.

62 Ebenda, S. 361-362.

63 BVerfGE 126, 286, S. 307 - Honeywell: ,allerdings wiegen hier Kompetenzüberschreitungen besonders schwer".

64 BVerfGE 123, 267, S. 359 - Lissabon. 
Lissabon-Urteil festgeschrieben. Deshalb musste die Bundesregierung den Europäischen Rat überzeugen, den Umbau der WWU nicht länger eigenmächtig abseits der Verträge zu betreiben. Das Gebot der verfassungsnormativen - und textförmlichen ${ }^{65}$ - Vorsorge für den Not- und Krisenfall gilt auch für das abgeleitete Verfassungsrecht der Europäischen Union. Der Vertrag bindet die Union an das Prinzip der begrenzten Einzelermächtigung. Unionsorgane können sich nicht selbst ermächtigen, auch nicht im finanzpolitischen Notstand. Und die Herren der Verträge sind ihrerseits an das Recht der Vertragsänderung gebunden, deshalb nicht frei, die WWU-Baustatik auf Dauer mit einem Schwarzbau außerhalb der Verträge zu stabilisieren. Die Mitgliedstaaten der Eurozone waren und bleiben verpflichtet, der Brückenkonstruktion EFSM/ EFSF ein festes Fundament zu geben. Der projektierte Art. 136 Abs. 3 AEUV fungiert als Schutzschirm für den Rettungsschirm: Niedergelegt im Vertragstext schützt der unionsrechtliche Solidaritätsimperativ den ESM - und sich selbst vor dem Ultra-vires-Einwand.

\section{Der ESM und der „,Kerngehalt der Verfassungsidentität“}

Wenn aber die staatlichen Integrationsgesetzgeber dem finanzpolitischen Rütlischwur beitreten, etablieren sie Krisenreaktionsrecht nicht nur in der Unionsverfassung, sondern auch in den Staatsverfassungen. Art. 23 GG erfasst den Umbau der WWU als Verfassungsänderung ohne Übertragung von Hoheitsrechten. Sie lässt sich ins Werk setzen nur mit Zweidrittelmehrheiten in Bundestag und Bundesrat. ${ }^{66}$ Die Vertragsstaaten öffnen die Unionsverfassung für Sonderrecht in der Eurozone, ${ }^{67}$ für ein Recht der Nothilfe, das im Krisenfall auch das staatliche Haushalts- und Verschuldungsverfassungsrecht durchbricht ${ }^{68}$ - und die Intensität der Verfassungsdurchbrechung steigert, sobald sich die Gruppe der Sicherungsgeber verkleinert und die der , stepping-out guarantors ‘ vergrößert. Derzeit allenfalls denkbare Eskalationsszenarien nehmen außer Griechenland, Irland und Portugal noch Spanien und Italien als Nehmerstaaten in den Blick. ${ }^{69}$ Freilich, auch das undenkbare Szenario gibt zu denken: Hülfe uns der Katalog von Karlsruhe, wenn Deutschland in Finanzierungsnot geriete und Austerität schuldete? Hielten wir für diesen Fall mit der gleichen Beflissenheit die souveräne Gesamtverantwortung des Bundestages über Einnahmen und Ausgaben fest?

Fragen wir lieber aus Sicht einer bürgschaftspflichtigen Bundesrepublik nach der Vereinbarkeit des Rütlischwurs mit Art. 79 Abs. 3 GG. Noch sehen wir ,Deutschland als vermutlichen Hauptfinanzierer von künftigen Hilfsleistungen“. ${ }^{70}$ Damit ist der Katalog von Karlsruhe angesprochen als letztes demokratisches Bollwerk gegen die Bereitschaft des verfassungsändernden Integrationsgesetzgebers, deutsche Finanzkraft in ein europäisches, bündisch-solidarisches Nothilfekonzept einzubringen. Wohlgemerkt: In dieser Konstellation

65 Vgl. Art. 79 Abs. $1 \mathrm{GG}$.

66 Art. 23 Abs. 1 Satz 3 GG - in der Variante der „,ermöglicht[en]“ Änderung des Grundgesetzes - in Verbindung mit Art. 79 Abs. 2 GG. Im Ergebnis wie hier Christian Calliess: Das europäische Solidaritätsprinzip und die Krise des Euro - Von der Rechtsgemeinschaft zur Solidaritätsgemeinschaft?, S. 63-64, abrufbar unter: http:// www.polsoz.fu-berlin.de/en/v/transformeurope/publications/latest_publications/authors/HU_FCE_Rede_Calli ess.pdf (letzter Zugriff: 30.06.2011). Anders dagegen die Bundesregierung: ,mit einfacher Mehrheit“. Vgl. Antwort des Parlamentarischen Staatssekretärs Steffen Kampeter vom 24. Februar, in: Deutscher Bundestag: Schriftliche Fragen mit den in der Woche vom 21. Februar 2011 eingegangenen Antworten der Bundesregierung, Bundestagsdrucksache 17/4915, S. 12.

67 Europäischer Rat: 24./25. März 2011, S. 21: ,daneben können sich auch Mitgliedstaaten, die dem Euro-Währungsgebiet nicht angehören, auf Ad-hoc-Basis beteiligen“.

68 Siehe S. 122-123 in diesem Band.

69 Dirk Meyer: Stabilitätsgefahren für die EWU, in: Wirtschaftsdienst 12/2010, S. 805-813, hier S. 807, mit systematischer Kalkulation verschiedener Ausfallszenarien.

70 Axel Weber/Jens Ulbrich/Karsten Wendorff: Krisenhilfe ohne Gemeinschaftshaftung, in: Frankfurter Allgemeine Zeitung, 03.03.2011. 
geht es nicht mehr um Übereinstimmung mit dem Haushalts- und Schuldenverfassungsrecht der Art. 109, 115 GG ${ }^{71}$ sondern um eine andere, qua Verfassungsänderung europäisierte Finanzverfassung und deren Vereinbarkeit mit der letzten Maßstabsnorm, die den Verfassungsgesetzgeber bindet. „Eine das Demokratieprinzip und das Wahlrecht zum Deutschen Bundestag in seinem substantiellen Bestimmungsgehalt verletzende Übertragung des Budgetrechts des Bundestages läge vor, wenn die Festlegung über Art und Höhe der den Bürger treffenden Abgaben in wesentlichem Umfang supranationalisiert würde. "72

Der neue Art. 136 Abs. 3 AEUV und der ESM dürfen und wollen gerade nicht ,in wesentlichem Umfang“ supranationalisieren. Freilich, erst recht gegen einen hybriden, zwischenstaatlich konstruierten, in der Zielrichtung aber integrationssolidarischen ESM muss der Bundestag die parlamentarische Budgethoheit verteidigen. Der Integrationsgesetzgeber muss die Supranationalisierungssperre ${ }^{73}$ in das operative Geschäft des ESM hinein verlängern und eine Quasi-Vergemeinschaftung auf zweiter Ebene verhindern. Das Grundgesetz verbietet die Entparlamentarisierung des Europäischen Stabilitätsmechanismus. Der ESM darf die traditionsreiche Budgethoheit des Parlaments ${ }^{74}$ nicht aufbrechen. Bundestag und Bundesrat dürfen nicht als Integrationsgesetzgeber den Haushaltsgesetzgeber und damit sich selbst entmachten, sie dürfen nicht die Einzelfallentscheidung über konkreten ESS vorbehaltlos den Regierungen der Eurozone überantworten. Hier drängt sich unter kompetenzrechtlichen Vorzeichen ein Vergleich mit dem wehrverfassungsrechtlichen Parlamentsvorbehalt auf, der zu einem verfassungsrechtlichen Analogieschluss führt. Zwar geht es nicht um Leben und Tod, aber immerhin um Wohl und Wehe der Steuerzahler in Deutschland. ${ }^{75}$

\section{Parlamentsvorbehalt im System gegenseitiger kollektiver Sicherheit}

Der haushaltsrechtliche Parlamentsvorbehalt hat verfassungsrechtlich eine vergleichbar starke Kraft wie der wehrverfassungsrechtliche. Dieser bleibt „,integrationsfest“ ${ }^{76}$ solange die Bundeswehr existiert, nicht eingeschmolzen wird in einer europäischen Armee; über den Einsatz deutscher Soldaten entscheidet der Deutsche Bundestag. Der ESM gleicht dem „System gegenseitiger kollektiver Sicherheit“ des Art. 24 Abs. 2 GG: ,jeder Staat soll gleichzeitig Garant und Garantieempfänger sein“. ${ }^{77}$ Die konstitutive Entscheidung über jeden einzelnen Kampf- beziehungsweise Finanzhilfeeinsatz obliegt dem Parlament. Die Bundeswehr ist „Parlamentsheer“78 - der Bundeshaushalt Parlamentsbudget. Der materiellen Legitimationsgrundlage des Art. 24 Abs. 2 GG („Wahrung des Friedens“) entspricht für das

71 Deren Maßstäblichkeit steht zur Diskussion, wenn der einfache Gesetzgeber den Bundesminister der Finanzen ermächtigt, Gewährleistungen für Verbindlichkeiten zu übernehmen. Vgl. Kurt Faßbender: Der europäische ,Stabilisierungsmechanismus“ im Lichte von Unionsrecht und deutschem Verfassungsrecht, in: Neue Zeitschrift für Verwaltungsrecht 13/2010, S. 799-803; Hanno Kube/Ekkehart Reimer: Grenzen des Europäischen Stabilisierungsmechanismus, in: Neue Juristische Wochenschrift 27/2010, S. 1911-1916; Monika Polzin: Finanzhilfen für Griechenland: Verfassungsrechtliche Schranken?, in: Die Öffentliche Verwaltung 6/2011, S. 209-215.

72 BVerfGE 123, 267, S. 361 - Lissabon.

73 Art. 48 Abs. 6 UAbs. 3 EUV.

74 „Schlußstein einer Parlamentarisierung des konstitutionellen staatsrechtlichen Systems“, so Christian Waldhoff: Grundzüge des Finanzrechts des Grundgesetzes, in: Josef Isensee/Paul Kirchhof (Hrsg.): Handbuch des Staatsrechts der Bundesrepublik Deutschland, Band V, Heidelberg 2007, § 116 Rdz. 122; Josef Isensee: Budgetrecht des Parlaments zwischen Schein und Sein, in: Juristenzeitung 20/2005, S. 971-981.

75 Die Gemeinschaft der Steuerzahler in Deutschland ist nicht identisch mit der Gemeinschaft der Staatsbürger: Unbeschränkt steuerpflichtig sind die Ansässigen (grundsätzlich mit ihrem Welteinkommen) ungeachtet ihrer Staatsangehörigkeit, beschränkt steuerpflichtig sind alle Gebietsfremden mit inländischen Einkünften. Vgl. $\S \S 1,49$ Einkommensteuergesetz und $\S \S 1,2$ Körperschaftsteuergesetz.

76 BVerfGE 123, 267, S. 361 - Lissabon.

77 BVerfGE 90, 286, S. 348-349 - AWACS I.

78 Ebenda, S. 382. 
Sicherheitssystem des ESM Art. 23 Abs. 1 GG, das Staatsziel des ,vereinten Europas“, gerichtet auf „Solidarität zwischen den Mitgliedstaaten“. ${ }^{79}$ „Allerdings klärt die in Art. 24 Abs. 2 GG enthaltene materielle Legitimationsgrundlage nicht die Frage, wer im innerstaatlichen Bereich von Verfassungs wegen über solche Einsätze zu entscheiden hat. " 80 Das Wehrverfassungsrecht gibt die Antwort ,,vor dem Hintergrund der deutschen Verfassungstradition“ im Rekurs auf „,ein allgemeines Prinzip“, „,nach dem jeder Einsatz bewaffneter Streitkräfte der konstitutiven, grundsätzlich vorherigen Zustimmung des Deutschen Bundestages bedarf". ${ }^{81}$ Das Haushaltsverfassungsrecht kann schwerlich eine andere Antwort geben. Denn der Gouverneursrat im ESM greift auf Einsatzkapazitäten nicht im EU-Haushalt, sondern in den Budgets der Mitgliedstaaten zurück.

„Der Einsatz bewaffneter Gewalt bedeutet nicht nur ein erhebliches Risiko für Leben und Gesundheit deutscher Soldaten, sondern er birgt auch ein politisches Eskalations- oder doch Verstrickungspotential." ${ }^{82}$ Nicht minder birgt europäische Finanzpolitik ein Eskalationsund Verstrickungspotential: solange die Staatshaushalte nicht auf Kante genäht werden, sondern aus allen Nähten platzen; solange die Zahl der ,stepping-out guarantors ' zunimmt; solange populistische Kräfte mobilisieren können mit ,bail out'-Schreckensszenarien. Der haushaltsverfassungsrechtliche Parlamentsvorbehalt sichert die adäquate Organzuständigkeit für jeden Fall der Finanzhilfe. Die vorherige Generalermächtigung aus Art. 115 Abs. 1 GG für „,die Übernahme von Bürgschaften, Garantien oder sonstigen Gewährleistungen“ bietet keinen Ersatz für Haushaltsplanung im Zeichen der Vollständigkeit und Einheit. ${ }^{83}$ Das Parlament muss das Abstimmungsverhalten des Bundesfinanzministers im Gouverneursrat des ESM in den wesentlichen Entscheidungen ${ }^{84}$ mandatieren.

Der Reichstag der Weimarer Republik stellte die dem Reich aufgebürdeten Reparationslasten als ,durchaus zwangsläufig“ 85 in seine Haushaltsplanung ein, als unabweisbare Verbindlichkeiten aus internationalen Verträgen. ${ }^{86}$ Dagegen hat die Finanzhilfe im ESM einen historisch unvergleichlich anderen Charakter. Sie ist keine aufgebürdete Last, aber auch „keine gütige Samariterleistung“, ${ }^{87}$ sondern Einstandsleistung in bündischer Gegenseitigkeit. Rückhalt für budgetwirksame Solidarität im Gemeinschaftsinteresse wird nur auf hohem Legitimationsniveau zu gewinnen sein, also parlamentarisch. Mit der einmaligen $\mathrm{Zu}-$ stimmung $\mathrm{zu}$ einem neuen Art. 136 Abs. 3 AEUV ist es nicht getan. Wegen der Unbestimmtheit und verfassungsrechtlichen Tragweite dieser Integrationsermächtigung erstreckt sich die Verantwortung des Parlaments auf den weiteren Vertragsvollzug. ${ }^{88}$

\section{Darf die Vertragsänderung scheitern?}

Frankreichs Wirtschafts- und Finanzministerin Christine Lagarde erklärte Ende 2010 in der Süddeutschen Zeitung: ,Im EU-Vertrag von Lissabon steht: Ein EU-Staat darf einem anderen EU-Staat, der sich in finanziellen Schwierigkeiten befindet, nicht helfen. Doch der Griechenland-Rettungsplan führt genau dazu. Auch der Euro-Rettungsschirm ist im Lissa-

79 Art. 3 Abs. 3 UAbs. 3 EUV.

80 BVerfGE 121, 135, S. 157 - AWACS II.

81 Ebenda. S. 154.

82 Ebenda, S. 161.

83 Art. 110 Abs. 1 GG

84 Europäischer Rat: 24./25. März 2011, Anlage II, S. 21-23.

85 Verhandlungen des Reichstages, Wahlperiode 1924/28, Nr. 3854, Haushaltsentwurf 1928, S. 8.

86 Max E. F. Kühnemann: Haushaltsrecht und Reichsetat, Berlin 1930, S. 112-113.

87 Peter-Christian Müller-Graff: Einander ausgeliefert, in: Frankfurter Allgemeine Zeitung, 20.05.2010.

88 Vgl. BVerfGE 121, 135, S. 157-163 - AWACS II; BVerfGE 123, 267, S. 434-435 - Lissabon. 
bon-Vertrag nicht vorgesehen. Trotzdem haben wir ein umfassendes Rettungssystem geschaffen - und sind dafür über die bestehenden Regeln hinaus gegangen." ${ }^{\text {"89 }}$

Der Trotz gibt zu denken. Wird hier unverhohlen einem Vertragsbruch das Wort geredet? Wer mit dieser Entschlossenheit die Differenz zwischen Können und Dürfen überspielt, muss gute Gründe haben. Die EFSM-Verordnung vom 11. Mai 2010 spricht sie aus: „Wird in dieser Situation nicht umgehend gehandelt, könnten Stabilität, Einheit und Integrität der Europäischen Union insgesamt ernsthaft bedroht sein. "90 Geht es um jene Not, die kein Gebot kennt, womöglich auch die Herren der Unionsverträge freizeichnet, oder in Wahrheit um „die nicht legitimierte Geschäftsgrundlage einer Transferunion“??91

\section{Das EFSM/EFSF-Provisorium: antizipierte Verfassungsänderung ...}

Verfassungsrecht muss um seiner selbst willen auch und gerade den Notstand für möglich halten und regulieren - zur Behauptung seines Anspruchs, unbestrittene Grundordnung der Rechtlichkeit zu sein, zumal Hoheitsgewalt ins Recht zu setzen. Art. 122 Abs. 2 AEUV weiß ,,von Naturkatastrophen oder außergewöhnlichen Ereignissen“ und verheißt dem katastrophal betroffenen Mitgliedstaat „einen finanziellen Beistand der Union“. Auf diese Rechtsgrundlage hat der Rat seine EFSM-Verordnung gestützt. Der EFSM freilich konnte die erwünschten Volumina nicht gewährleisten. Der Beistand „,der Union“ bleibt mit potenziell 60 Milliarden Euro quantitativ deutlich zurück hinter dem EFSF-Beistand ,der Staaten“ mit bis zu 500 Milliarden Euro. Damit zehrt die EFSM/EFSF-Konstruktion nicht allein vom EU-Haushalt, sondern wesentlich von den Budgets der Mitgliedstaaten der Eurozone. Erstreckt in die staatliche Finanzverfassung, ${ }^{92}$ erweisen sich bereits die ,Notmaßnahmen " als camouflierte Verfassungsänderung, als freihändiger Vorgriff im Umbau der WWU und gesamthänderischer Zugriff auf einen virtuellen Euro-Staaten-Haushalt ${ }^{93}$ - eine Verschiebung der Statik, die ersichtlich nicht nur die Unionsverfassung verändert. Der neue Solidaritätsimperativ in der Eurozone kann als Umprägung ihrer Gesamtverfassung gelten, vor allem in der bündischen Horizontalbeziehung der Mitgliedstaaten der Eurozone untereinander. ${ }^{94}$

Dass die neue WWU auf Dauer Rückhalt finden könnte in einem Provisorium der akuten Krise, liegt fern. Noch ferner liegt nur die These, Deutschland könne sich an der EFSFZweckgesellschaft ohne gesetzliche Ermächtigungsgrundlage beteiligen. ${ }^{95}$ Denn die Beteili-

89 Michael Kläsgen/Stefan Ulrich: „Wir werden bedingungslos sparen“. Interview mit Christine Lagarde, in: Süddeutsche Zeitung, 23.12.2011. Gemeint sind nicht EU-Staaten, sondern Euro-Staaten. Für EU-Staaten, die nicht der Eurozone angehören, kann im Wege des Art. 143 AEUV „Beistand“ mobilisiert werden; vgl. die Entscheidung des Rates vom 4. November 2008 über einen mittelfristigen finanziellen Beistand der Gemeinschaft für Ungarn, in: Amtsblatt der EU, Nr. L 37 vom 6. Februar 2009, S. 5-6.

90 Erwägungsgrund 4 EFSM-VO.

91 Dirk Meyer: Zur juristischen Begründung des ,Rettungsschirms‘, in: Jahrbuch für Wirtschaftswissenschaften, Band 61, Göttingen 2010, S. 103-124, hier S. 121.

$92 \S 1$ Abs. 1 Satz 1 des Gesetzes zur Übernahme von Gewährleistungen im Rahmen eines europäischen Stabilisierungsmechanismus (StabMechG) vom 22. Mai 2010, BGB1. I S. 627: „Das Bundesministerium der Finanzen wird ermächtigt, für Kredite, die eine von den Mitgliedstaaten des Euro-Währungsgebietes gegründete oder beauftragte Zweckgesellschaft zur Finanzierung von Notmaßnahmen zum Erhalt der Zahlungsfähigkeit eines Mitgliedstaates des Euro-Währungsgebietes aufnimmt, Gewährleistungen bis zur Höhe von insgesamt 123 Milliarden Euro zu übernehmen [...]".

93 Der Schlüssel für die Zeichnung des EZB-Kapitals bestimmt die Aufteilung der finanziellen Beiträge zum ESM: Auf Deutschland entfallen mindestens 27,146 Prozent, auf Frankreich 20,386 Prozent, dann folgen Italien (17,914 Prozent) und Spanien (11,904 Prozent). Vgl. Europäischer Rat: 24./25. März 2011, Anlage II, S. 34.

94 Vgl. Hufeld: Die Verfassungsdurchbrechung, 1997, S. 15-25, 208-230 zur Rechtfertigung(-snot) der Verfassungsdurchbrechung; Mussler/Seiser: EU schließt Umschuldung Griechenlands aus, 2011.

95 So Joachim Wieland: Der Rettungsschirm für Irland, in: Neue Zeitschrift für Verwaltungsrecht 6/2011, S. 340343, hier S. 341, mit Verweis auf $§ 65$ Bundeshaushaltsordnung. 
gung Deutschlands an der luxemburgischen Societé Anonyme (Firma: EFSF) lässt sich allenfalls halten - rückwirkend heilen - als vorgreifliche, provisorisch-notrechtlich antizipierte Verfassungsänderung. Verfassungsrevision jedoch ist die vornehmste Aufgabe der Legislative. Wenn die Revision darauf zielt, ,,die Finanzstabilität in der Währungsunion sicherzustellen“" ${ }^{96}$ und mit einer Änderung der Unionsverfassung zusammenfällt, geht es um parlamentarische Verfassungsgesetzgebungsverantwortung (Integrationsverantwortung) auf zwei Ebenen, um Integration durch Revision und Revision durch Integration. ${ }^{97}$

Wenn die Ergänzung des Art. 136 AEUV gelingt, wächst dem EFSM/EFSF-Provisorium nachträglich Legitimation zu. Das Provisorium trägt als Notbrücke aus der alten in die neue WWU, als ,Maßnahme“ zur Ergänzung des jedenfalls auf der Rechtsfolgenseite überforderten Art. 122 Abs. 2 AEUV: Zur Bewältigung der gegenwärtigen Krise reicht die Finanzmacht der Union nicht aus. Zudem überbrückt das Provisorium die schiere Unmöglichkeit einer schnellen Vertragsänderung. Wenn diese aber zeitverzögert zustande kommt, sanktioniert sie rückwirkend die Entschlossenheit der Mitgliedstaaten, für die Währungsunion einzustehen; wenn für Deutschland der verfassungsändernde Integrationsgesetzgeber zustimmt, so billigt er nachträglich auch die einfach-gesetzliche Notlösung im Übergang.

\section{... oder haltlose Notpolitik}

Josef Isensee hat vermerkt, dass ,hoffnungslos einseitiger Transfer auf Dauer den staatlichen Zusammenhalt gefährden kann“.$^{98}$ Das gilt erst recht für den nicht-staatlichen Zusammenhalt in der Union. Die ESM-Logik - Kredit gegen Wohlverhalten - will die Transferunion verhindern, sie will keinen Länderfinanzausgleich nach deutschem Muster. Dennoch muss die Rettungsschirm-Politik jenen Vorwurf der Einseitigkeit fürchten, Grenzen der Solidarisierungsbereitschaft, noch dazu politischen Widerstand in den Kreditnehmerstaaten, wenn dort die Austeritätsverpflichtung als finanzpolitisches Diktat verunglimpft wird. „Derartige Aktionen strapazieren den Zusammenhalt der EU insgesamt. “99 Alles spricht dafür, dem Solidaritätsmechanismus qua Vertragsänderung eine unangreifbare Legitimationsgrundlage zu geben. Die Herren der Verträge betreten eine schiefe Bahn, wenn sie ein Notrecht auf Verfassungsdurchbrechung praeter constitutionem postulieren. ${ }^{100}$

Die außervertragliche Notrettung wird nachträglich Halt finden im vertraglichen Rütlischwur ${ }^{101}$ oder haltlos sich selbst delegitimieren. Wenn die Rückversicherung im Vertrag scheitert, kehren sich Misserfolg und Legitimationsverweigerung auch gegen das heutige EFSM/EFSF-Provisorium. Dann hätte sich die Rettungsschirm-Politik als Notpolitik erwiesen, die auf Zustimmung nicht rechnen darf. Dann bleibt nur mehr die Hoffnung, dass die Eurozone weitere Belastungsproben auf Solidarität vermeidet, jeder Teilnehmer sich selbst helfen kann. Dieses Euroland wäre Schicksalsgemeinschaft ohne Rütlischwur.

$96 \S 1$ Abs. 1 Satz 1 StabMechG.

97 Hufeld: Europäische Integration und Verfassungsänderung, 2011, 1. Abschnitt, Rdz. 3-31.

98 Josef Isensee: Idee und Gestalt des Föderalismus im Grundgesetz, in: Josef Isensee/Paul Kirchhof (Hrsg.): Handbuch des Staatsrechts der Bundesrepublik Deutschland, Band VI, Heidelberg 2008, § 126, Rdz. 159.

99 Müller-Graff: Einander ausgeliefert, 2010.

100 Dazu eingehend und mit Recht ablehnend Thomas Giegerich: Europäische Verfassung und deutsche Verfassung im transnationalen Konstitutionalisierungsprozeß, Berlin u.a. 2003, S. 523-573.

101 Beurkundet in Art. 136 Abs. 3 AEUV. 